ACCEPTED MANUSCRIPT • OPEN ACCESS

\title{
Investigating thermal stability of metallic and non-metallic nanoparticles using novel graphene oxide-based transmission electron microscopy heating-membrane
}

To cite this article before publication: Nitin M. Batra et al 2022 Nanotechnology in press https://doi.org/10.1088/1361-6528/ac547c

\section{Manuscript version: Accepted Manuscript}

Accepted Manuscript is "the version of the article accepted for publication including all changes made as a result of the peer review process, and which may also include the addition to the article by IOP Publishing of a header, an article ID, a cover sheet and/or an 'Accepted Manuscript' watermark, but excluding any other editing, typesetting or other changes made by IOP Publishing and/or its licensors"

This Accepted Manuscript is $\odot 2022$ The Author(s). Published by IOP Publishing Ltd..

As the Version of Record of this article is going to be / has been published on a gold open access basis under a CC BY 3.0 licence, this Accepted Manuscript is available for reuse under a CC BY 3.0 licence immediately.

Everyone is permitted to use all or part of the original content in this article, provided that they adhere to all the terms of the licence https://creativecommons.org/licences/by/3.0

Although reasonable endeavours have been taken to obtain all necessary permissions from third parties to include their copyrighted content within this article, their full citation and copyright line may not be present in this Accepted Manuscript version. Before using any content from this article, please refer to the Version of Record on IOPscience once published for full citation and copyright details, as permissions may be required. All third party content is fully copyright protected and is not published on a gold open access basis under a CC BY licence, unless that is specifically stated in the figure caption in the Version of Record.

View the article online for updates and enhancements. 


\title{
Investigating thermal stability of metallic and non-metallic nanoparticles using novel graphene oxide-based transmission electron microscopy heating-membrane
}

\author{
Nitin M. Batra ${ }^{1,4}$, Dinesh K. Mahalingam ${ }^{2}$, Pradeep Doggali ${ }^{3}$, Suzana P. Nunes ${ }^{2}$, Pedo M.F.J. \\ Costa ${ }^{1, *}$ \\ ${ }^{1}$ Physical Science and Engineering (PSE) Division, King Abdullah University of Science and \\ Technology (KAUST), Thuwal, Kingdom of Saudi Arabia, 23955-6900. \\ ${ }^{2}$ Biological and Environmental Science and Engineering (BESE) Division, King Abdullah \\ University of Science and Technology, Thuwal, Kingdom of Saudi Arabia, 23955-6900. \\ ${ }^{3}$ KAUST Catalyst Center (KCC), King Abdullah University of Science and Technology, Thuwal, \\ Kingdom of Saudi Arabia, 23955-6900. \\ ${ }^{4}$ Institut Des Materiaux Jean Rouxel, CNRS-University of Nantes, Nantes, 44300, France. \\ * nitinkumar.batra@kaust.edu.sa, pedro.dacosta@kaust.edu.sa
}

Keywords: in-situ TEM, Graphene oxide heater, Gold, Silica, KCC-1, graphene etching, carbon shell

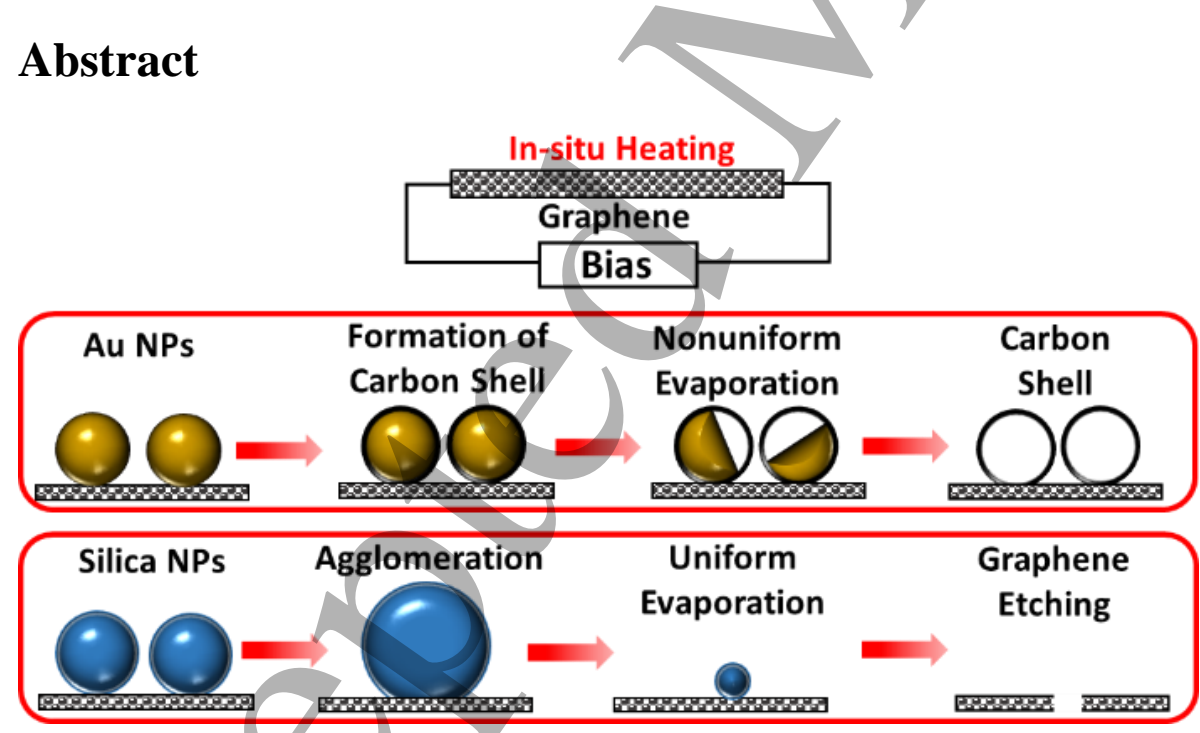

In recent years, graphene has been explored as a heating membrane for studying high-temperature dynamics inside the transmission electron microscope (TEM) due to several limitations with the existing silicon nitride-based membrane. However, the transfer of monolayer CVD graphene for TEM experiments is challenging and requires many complicated steps with a minimum success rate. This work developed a novel in-situ heating platform by combining the graphene oxide (GO) flakes in the pre-patterned chips. The isolated GO flake was self-suspended between the metal electrodes by a simple drop-casting process. The GO was reduced and characterized using Raman and electron energy-loss spectroscopy. Furthermore, a GO-based heater was used to investigate 
the thermal stability of gold and silica nanoparticles. Conversely, the gold nanoparticles evaporated non-uniformly and left an empty carbon shell, while silica disappeared uniformly by etching carbon support. We successfully demonstrated a GO flake as a heating membrane to study high temperature thermal dynamic reactions: melting/evaporation, agglomeration, Rayleigh instability, and formation/or removal of carbon in the nanoparticles.

\section{Introduction}

For studying dynamics reactions of nanomaterials at high temperatures, specially designed environmental transmission electron microscope (TEM) and in-situ TEM holders are commonly used. The in-situ TEM experiments directly visualize the various dynamic phenomena at the atomic resolution and help develop a clear understanding between the structure, morphology, and chemical nature of different materials. Owing to lower cost and ease of use, in-situ holders have gained popularity for investigating phase transformation, melting/evaporation, alloy formation, and materials degradation reactions under various stimulus conditions. [1-8] The in-situ holders use specifically engineered silicon nitride membrane-based chips.[9,10] However, these silicon nitride-based chips suffered several limitations: thicker support membrane, poor contract, hightemperature instability, thermal drift, and membrane degradation above $>1100{ }^{\circ} \mathrm{C} .[11,12]$

Graphene, an electron transparent and atomically thin material, is the ideal candidate for TEM support film. Atomically resolved images are acquired from nanomaterials supported on the graphene under static and dynamic conditions.[13-15] However, several challenges are associated with integrating graphene into TEM chips. Synthesis of graphene requires costly setups such as high-temperature chemical vapour deposition (CVD) furnaces and sophisticated gas supply systems. Furthermore, optimization of growth conditions is a time and resource-consuming process. The successful growth of graphene does not guarantee its effective integration into devices. The final steps to place graphene on a target substrate are delicate, as the transfer steps involve various manual interventions and chemicals.[12,16,17] These factors prohibit its use in commercial heating chips.

We developed a low-cost and facile method for setting up a graphene oxide-based in-situ heater. Graphene oxide (GO), a graphene derivative, can be synthesized by chemical oxidation and exfoliation of graphite flakes to obtain different morphologies, sizes, and chemistries (oxygen functionalities).[19-21] In recent years, the applications of GO exploded ten-folds with the availability of scalable and easy synthesis methods, controllable morphologies, and chemistry of GO flakes. Moreover, the favourable chemicals groups on GO make it more hydrophilic, thus allowing GO dispersion to be more stable in water. In previous works, the graphitization of amorphous carbon and graphene oxide was achieved using current-induced annealing inside the vacuum.[14,18-22] In this work, the isolated GO flake was used as a support platform for suspending nanomaterials and studying dynamic heating experiments inside TEM. Using currentinduced Joule heating, the GO flake was reduced, and reduction was confirmed using Raman and EELS spectroscopy. Further, the thermal stability and high-temperature reactions of Au and silica nanoparticles supported on GO flake were examined at high resolution.

\section{Experimental Methods}

GO flakes were synthesized using a modified Hummers method, and the concentration of GO solution was optimized.[23,24] The GO dispersion diluted in water was used for these experiments without sonication. Diluted GO dispersion was drop-cast onto pre-fabricated in-situ TEM 
electrical chips.[25] Using an optical microscope, individual GO flakes suspended between electrodes were identified. Furthermore, the chips were transferred to Aduro Protochips holder and subsequently to the TEM. The whole setup was appropriately grounded to avoid failure of the GO flakes (due to parasitic static charges present in the system). In-situ experiments were performed on a Thermo Fisher Scientific Tecnai Twin TEM operating at low voltage (80-120 keV) under low dose conditions to reduce beam damage. A Gatan Orius charge-coupled detector (CCD) camera was used to record images and videos. Videos were captured using the Snagit screen recorder. Subsequently, the same chips were transferred to a Thermo Fisher Scientific image-corrected Titan TEM operating at $80 \mathrm{keV}$ to collect high-resolution micrographs and the electron energy loss spectrum (EELS). Raman spectra were collected from GO flakes using a WITEC Alpha300RA. The spectrum was acquired at a low dose $(0.5 \mathrm{~mW})$ with a laser source of $532 \mathrm{~nm}$ wavelength at 100x magnification. Scanning electron microscopy (SEM) image was collected with Thermo Fisher Scientific Nova Nano SEM, at 5 kV. A Park System XE-100 AFM was used to collect the topography and thickness details of the GO flake. The samples for AFM, SEM and Raman experiments were prepared by drop-casting $\mathrm{GO}$ dispersion onto a pre-clean $\mathrm{SiO}_{2} / \mathrm{Si}$ wafer.

Au nanoparticle (diameter: $15 \mathrm{~nm}$ ) dispersion was obtained from Aurion, The Netherlands. After that, Au particles were washed with water twice by centrifugation at 13,000 rpm (10 min), and subsequently, after each cycle, the sediments were re-dispersed in water. Silica nanoparticles (KCC-1) were prepared by modified microwave-assisted hydrothermal synthesis.[26] The silica nanoparticles (dispersed in ethanol) were drop-cast onto a TEM grid and GO membrane for TEM investigation.

\section{Results \\ Suspended graphene oxide-based chips:}

As-prepared stock-dispersion $(200 \mu \mathrm{g} / \mathrm{ml})$ was diluted to ultra-low concentration of $0.5 \mu \mathrm{g} / \mathrm{ml}$ in water (see Figure 1a). To prepare the suspended GO-based devices, one microliter of diluted dispersion of GO was pipetted onto the chips (inset image in Figure 1b) with a 3-5 $\mu$ m window and allowed to dry in air. As little dispersion was required for a GO device, we prepared tens of these devices with just a few microliters of diluted dispersion. The devices were inspected under an optical microscope to identify isolated GO flakes. The schematic setup of the GO-based heater is shown in Figure 1b. Between the trenches, unwanted GO flakes were easily removed by the laser source (of Raman instrument) at the high laser power due to oxidation of carbon atoms.[27] Then, the devices were transferred to the TEM column on a Protochips Aduro holder. TEM and SEM images of the GO-1 devices are shown in Figures 1c and 1d. Moreover, AFM and SEM images of as-produced GO flakes are provided in the Supplementary Information. After dropcasting the GO flakes on a blank wafer, we allowed it to dry in the fume-hood. Most of the GO flakes were flat. SEM images of as-synthesized GO flakes are shown in Figures S1a-1Sb. The AFM scan confirmed the planar morphology. Most flakes were more than three $\mu \mathrm{m}$ wide and 1.5$5 \mathrm{~nm}$ thick, corresponding to 1-4 graphene oxide layers as shown in the line profiles in Figures S1c-1Sd. More than $70 \%$ of flakes were less than $3 \pm 0.2 \mathrm{~nm}$ thick.

Electrical characterization of the GO flakes was performed in a two-probe configuration inside the ultrahigh vacuum of the TEM column without an electron beam irradiation. The electrical resistance of the prepared GO devices was of the order of a few $\mathrm{M} \Omega$ owing to the highly disordered structure and the presence of various functional groups.[20] In our initial setup, many GO devices 
failed due to residual electrical charges in the TEM holder (see in Figure S2). This issue was sorted out by grounding the holder and electrical supply unit before connecting the device to the holder. The resistance of the GO-1 device was around $23 \mathrm{M} \Omega$. More than ten devices were characterized, and most of these had resistance between 2-70 M $\Omega$. The GO flake was gradually heated by increasing the direct current that passed through it. Here, the ramping rate of $1-10 \mu \mathrm{A} / \mathrm{s}$ was used during the entire cycle. Within the first few seconds (as seen in Figure 1f), the resistance dropped more than $98 \%$ due to the Joule heating of GO and the metal-GO contacts.[28] Upon the currentinduced reduction of GO, the electrical conductivity of GO improved. I-V curve indicates the linear response of the GO flake before and after the reduction process (Figure 1e). The resistance was around $112 \mathrm{k} \Omega$ at $50 \mu \mathrm{A}$ current and dropped linearly with increasing current (see Figure 1f). When current was ramped gradually to $350 \mu \mathrm{A}$ and $650 \mu \mathrm{A}$, the resistance plummeted to $9.2 \mathrm{k} \Omega$ and $4.0 \mathrm{k} \Omega$, respectively. Additionally, three different devices with similar flake size were selected and subjected to current-induced annealing at $670 \mu \mathrm{A}, 1.0 \mathrm{~mA}$, and $1.2 \mathrm{~mA}$, respectively. The Raman and EELS spectrum of the following devices are presented in Figure 2. The EELS spectrum was collected in-situ, while for Raman the devices were analyzed ex-situ.

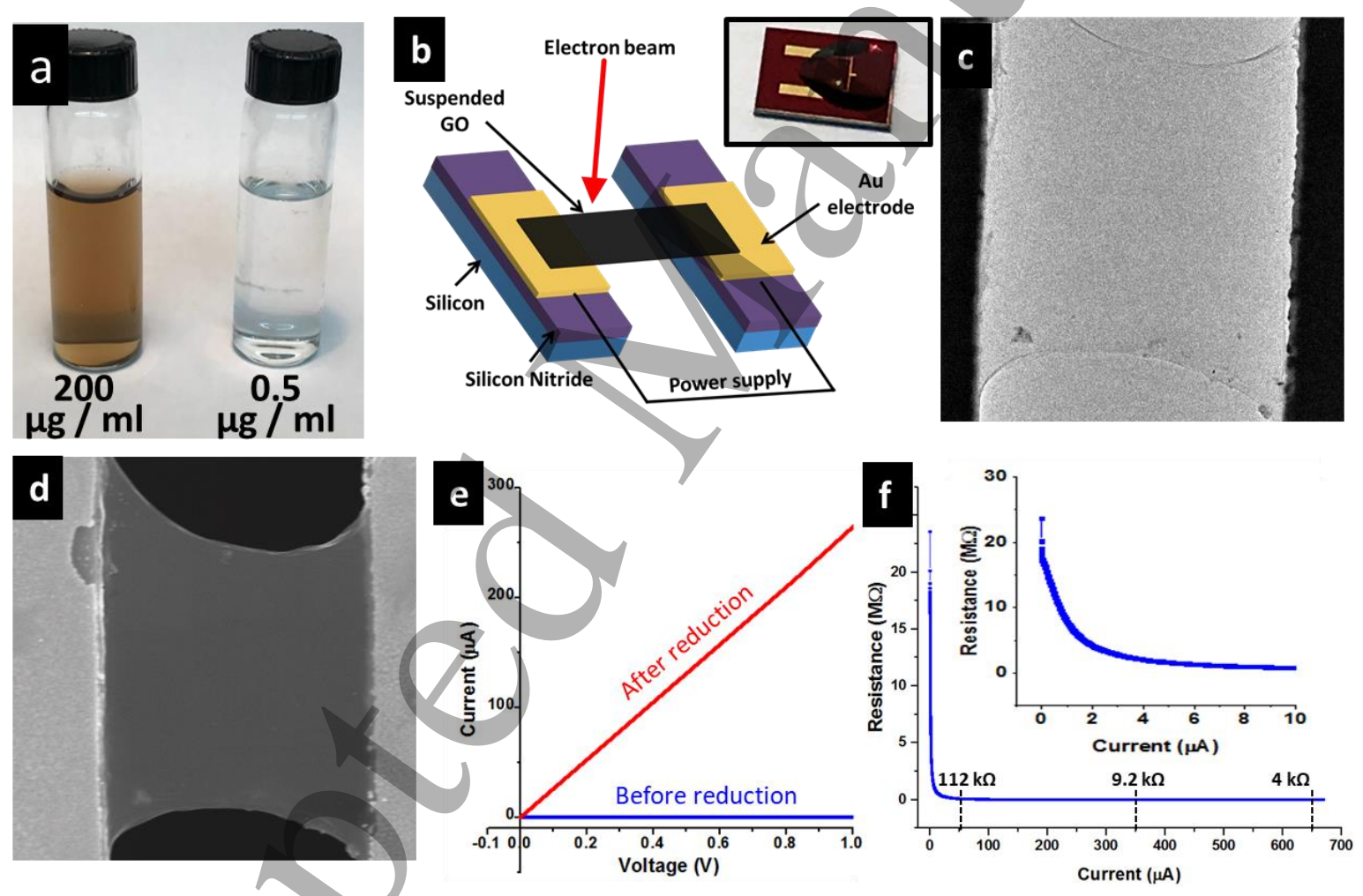

Figure 1 a) a photograph of vials with concentrated and diluted GO dispersion in water, b) schematic setup of the GO-based heater (heating is achieved by passing DC through the suspended flake), c) TEM, and d) SEM images of the GO-1 flake suspended between two electrodes, e) IV curve of the GO- 1 device before and after reduction, f) resistance vs. current curve during currentinduced reduction of the GO device. 

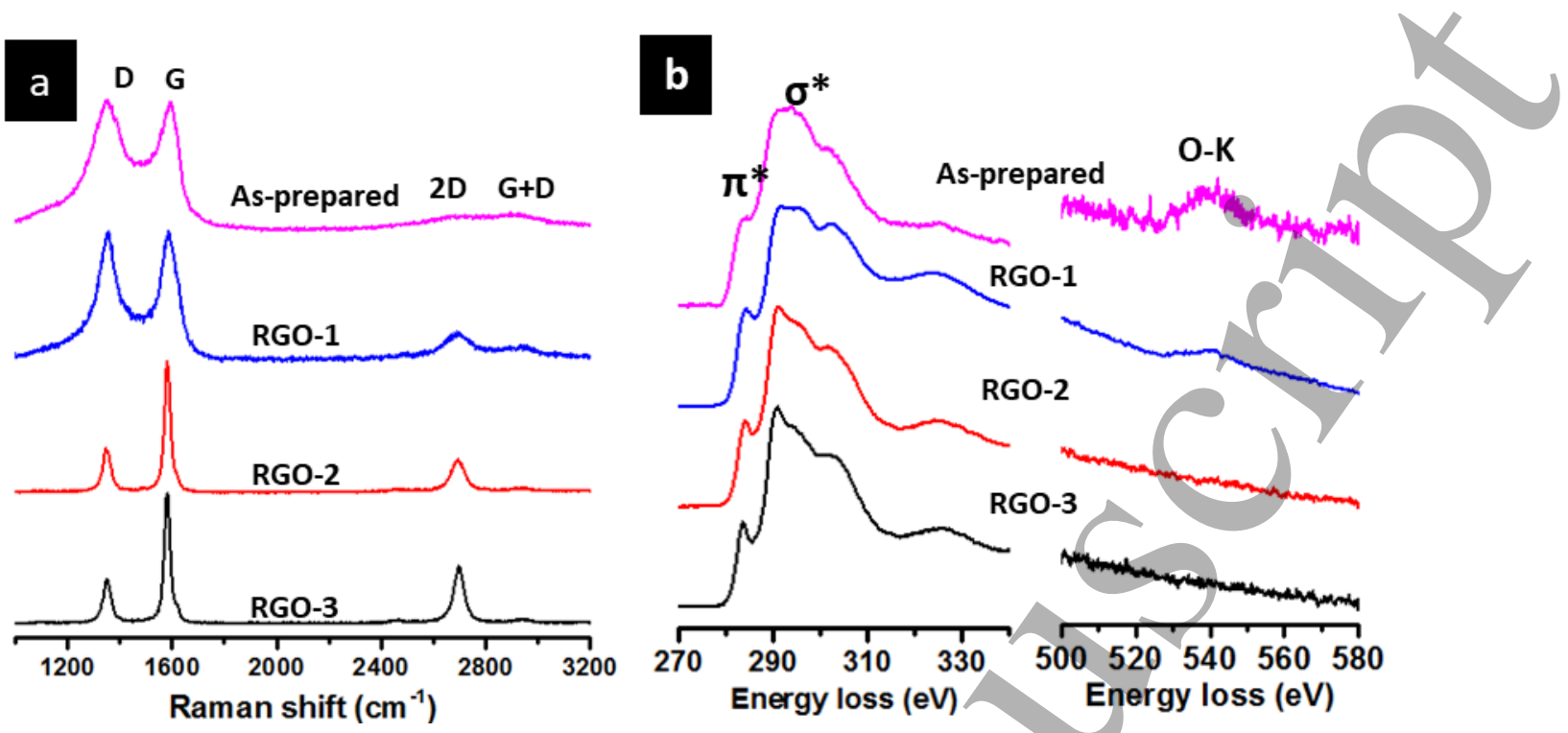

Figure 2 a) Raman spectra of GO and RGO-1, RGO-2, and RGO-3 , b) EELS spectra of GO and RGO-1, RGO-2, and RGO-3.

Raman spectra were collected from the suspended GO flake and three different reduced GO flakes (RGOs) (Figure 2a), representing characteristic G-, D- and 2D-peaks of carbon materials. Very low 2D and intense D peaks confirmed the highly disordered structure and the presence of various functional groups in the prepared GO device [30-33]. Furthermore, the G peak was also wide and was placed at $1597 \mathrm{~cm}^{-1}$. The degree of oxidation/reduction of GO flakes was accessed by calculating $\mathrm{ID}_{\mathrm{D}} / \mathrm{I}_{\mathrm{G}}$ ratio. The $\mathrm{ID}_{\mathrm{D}} / \mathrm{I}_{\mathrm{G}}$ ratios for $\mathrm{GO}$ and $\mathrm{RGOs}$ are shown in Table S1; the data from the literature of reduced GO flakes are also summarized. The $\mathrm{I}_{\mathrm{D}} / \mathrm{I}_{\mathrm{G}}$ intensity ratio of GO is higher than 1 , validating the presence of defects. RGO-1 device was reduced at a maximum current of $670 \mu \mathrm{A}$. Although the $\mathrm{ID}_{\mathrm{D}} / \mathrm{I}_{\mathrm{G}}$ of RGO-1 did not change significantly, the peak shift and the upturn in the intensity of the 2D peaks were observed. For samples RGO-2 and RGO-3, a higher amount of current was used for reduction. The higher the current and the higher the Joule heating temperature, the better the GO flake reduction is.[29] $\mathrm{I}_{\mathrm{D}} / \mathrm{I}_{\mathrm{G}}$ dropped significantly for RGO-2 and RGO-3 (see Table S1) due to the complete removal of functional groups and the improvement in the crystallinity of the graphene crystals.

Electron energy loss peaks around $284 \mathrm{eV}$ and $290 \mathrm{eV}$ are associated with the transition of electrons to empty $\pi^{*}$ states and empty $\sigma^{*}$ states, respectively, linked to carbon K-edge. The EELS can also access the chemistry and bonding of reduced GO, as reported in the literature.[18,19] The peak at $540 \mathrm{eV}$ is the oxygen $\mathrm{K}$-edge peak, associated with oxygen-rich functional groups present in GO. As seen in Figure $\mathbf{2 b}$, the shape and intensity of the carbon-K edge and oxygen K-edge peaks changed after the current-induced reduction of GO. $\pi^{*}$ peak roughly represents the content of $\mathrm{sp}^{2}$-bonded carbon atoms. The absence of $\pi^{*}$ peak and the appearance of an oxygen peak in the EELS spectrum validate the prepared GO flake's highly amorphous and oxygen-rich nature. As seen in Figure $2 \mathbf{b}$, the intensity of $\pi^{*}$ surged for RGO-1, RGO-2, and RGO-3. Higher $\pi^{*}$ intensity means more $\mathrm{sp}^{2}$-bonded carbon and better crystallinity. This also corroborates the intense $\mathrm{G}$ peak in Raman spectra of reduced flakes (Figure 2a). The intensity of the O-K peak was also reduced for RGO-1 compared to GO, confirming a partial reduction in GO. In comparison, the disappearance of O-K peak in RGO-2 and RGO-3 endorses the complete reduction of GO flake. For RGO-2 and RGO-3, the calculated current is density higher than $1.2 \times 10^{7} \mathrm{~A} / \mathrm{cm}^{2}$. Therefore, 
at this current density, the temperature of RGO flake was higher than $2000{ }^{\circ} \mathrm{C}$ due to Joule heating that confirmed the complete removal of oxygen and improved crystallization of graphene.[30,31]

\section{Gold nanoparticle thermal stability:}
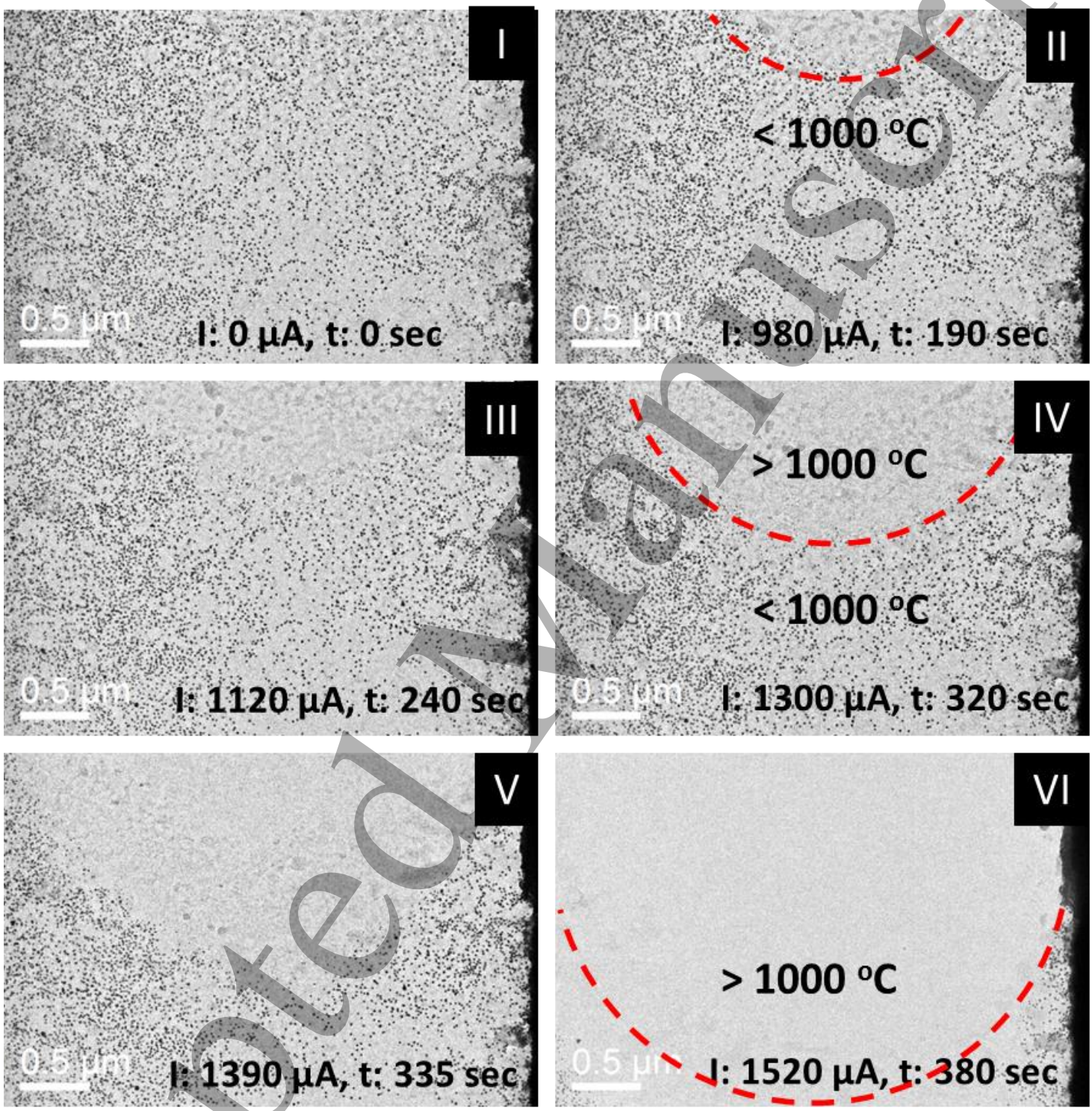

Figure 3 Time series TEM images of Au nanoparticles deposited on the RGO device. The images of the nanoparticle evaporation are labelled from panels I-VI sequentially. The red-dotted lines show the temperature distribution during the current-driven heating of the RGO device.

After the current-driven reduction of the suspended GO flakes, RGO-based heater devices were employed as a substrate to investigate the thermal stability of Au and silica nanoparticles. In the first case, the devices were prepared by drop-casting Au nanoparticles dispersion on the RGO chip. Figure 3 (panel I) shows the RGO flake decorated with Au nanoparticles (approximately $15 \mathrm{~nm}$ ). Further, joule heating of the Au-RGO system was carried out by passing a current through the flake. The temperature distribution was nonuniform, and a maximum temperature was observed at 
the centre of the flake. Moreover, the temperature profile was radial; the red-dotted lines in Figure 3 represent temperature boundaries. Previously, a similar heating profile was observed for graphene-based heaters.[14] Initially, the evaporation of particles was observed at the top of the TEM image (panels I and II in Figure 3). Some amorphous residues remained on the RGO flake after the Au nanoparticles evaporation in panels III and IV (see Figure 3). The residues might be coming from the sample preparation steps. However, both Au nanoparticles and amorphous residues disappeared when the current increased to $1520 \mu \mathrm{A}$ (current density: $0.6 \mathrm{x} 10^{7} \mathrm{~A} / \mathrm{cm} 2$ ). At this point, the temperature was higher than $1000{ }^{\circ} \mathrm{C}$ (panel VI, Figure 3) at the centre of the flake.

Moreover, we also directly heated Au nanoparticles on the same GO support before and after reduction, as shown in Figure S3. For the GO-Au nanoparticles system, Figures S3a and S3b display TEM images before and after evaporation of Au nanoparticles. Here, the reduction and heating coincided. Resistance vs. current curve of GO-Au nanoparticles system is shown in Figure S3c. Resistance plummeted from $8.1 \mathrm{M} \Omega$ to $2.4 \mathrm{k} \Omega$ due to in-situ reduction of GO. After the first $\mathrm{GO}$ reduction and $\mathrm{Au}$ evaporation cycle, the nanoparticles were re-deposited onto the same device. TEM images of the RGO-Au nanoparticle system are shown in Figure S3d. Only heating occurred during the second cycle, and the Au nanoparticles evaporated (Figure S3e). As observed in Figure S3f, no substantial resistance drop was observed for the RGO heater. GO and RGO heaters can be used for in situ thermal studies. However, RGO-based heaters were preferred due to fewer variations in resistance during experiments and the absence of functional groups. To identify the evaporation temperature of Au nanoparticles, ex-situ experiments were also performed by ex-situ heating of RGO-Au nanoparticle devices inside a vacuum-tube furnace. The devices were annealed at $9000^{\circ} \mathrm{C}, 950^{\circ} \mathrm{C}, 1000^{\circ} \mathrm{C}$, and $1050^{\circ} \mathrm{C}$ in a vacuum tube furnace $(>5 \mathrm{mTorr})$. As seen in Figures S4a-S4d, no significant changes were observed in the first RGO-Au nanoparticle device after treatment at $900{ }^{\circ} \mathrm{C}$ and $950{ }^{\circ} \mathrm{C}$. In the case of the annealed devices at $1000{ }^{\circ} \mathrm{C}$ (see Figures S4e and $\mathbf{S 4 f}$ ), the evaporation of most of the nanoparticles occurred except for a few large particles. While thermal annealing of the RGO-Au nanoparticle device at $1050{ }^{\circ} \mathrm{C}$ resulted in complete evaporation of Au nanoparticles, as seen in Figures S4g-S4h. 

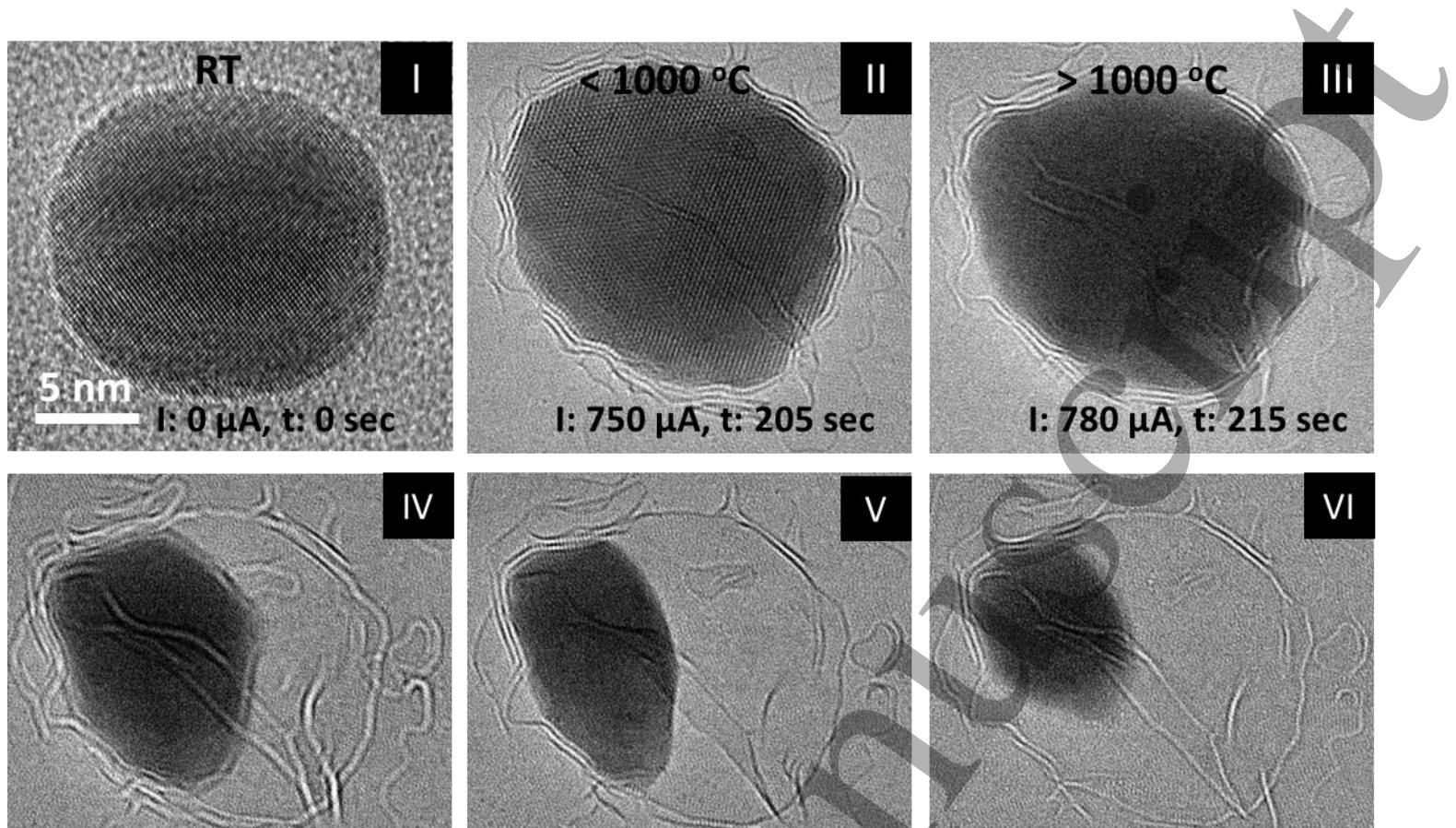

I: $810 \mu \mathrm{A}, \mathrm{t}: 235 \mathrm{sec}$
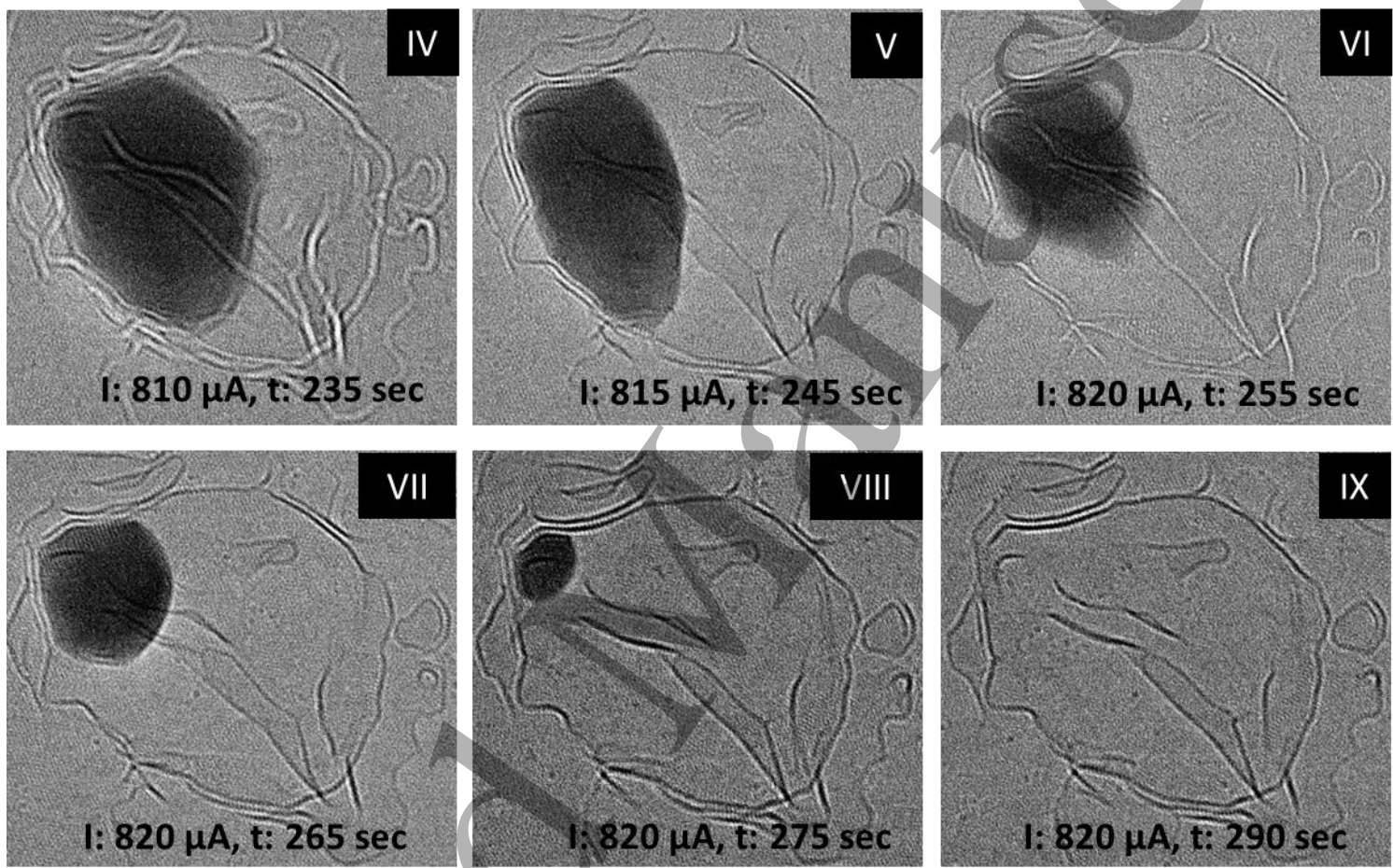

Figure 4 panels: I-IX: Time-series HRTEM images of the melting and evaporation of a single Au nanoparticle during the current ramping of the RGO support.

As seen in Figure 4 (panel I), Au nanoparticle with cubic crystal arrangement can be seen. In the as-received condition, no surface passivation layer was observed. When the RGO device was heated using Joule heating, both Au nanoparticles and RGO underwent structural rearrangement at a temperature close to the melting point of $\mathrm{Au}$. The orientation changes were also clear by comparing panels I and II of Figure 4 and their respective FFT images as shown in Figures S5a and S5b. Moreover, a thin carbon-nanoshell was also formed around the Au nanoparticle. Furthermore, from panels I to III in Figure 4, the Au nanoparticle underwent phase transformation from crystalline to disordered phase. No lattice fringes were noticed, and the shape of particles also changed. The previous report observed significant superheating in the nanoparticle covered with graphitic shells.[32] With the slight increase in temperature of the device, a significant reduction in the size of particles was noted (panel IV, Figure 4). This occurred due to possible evaporation of the surface $\mathrm{Au}$ atoms from the defect sights and broken carbon nanoshell. As seen from Figure 4 (panels IV to IX), particle size dropped slowly from 15 to a few nm before complete evaporation. Moreover, the evaporation of $\mathrm{Au}$ atoms was nonuniform. It was slower for $\mathrm{Au}$ atoms 
adjacent to carbon shells than atoms on free edges. Figure 4 (panel IX) shows the empty carbon nanoshell after the complete evaporation of the Au nanoparticle. Similar behavior was observed for almost all Au nanoparticles at lower magnification and doses, as seen in Figure S6. After panel IV in Figure S6, the experiment was stopped, and the remaining particles in the bottom left corner were analyzed. As seen in Figures S6b-c, some empty and partially filled carbon nanoshells were clearly observed.

\section{Silica nanoparticles thermal stability}
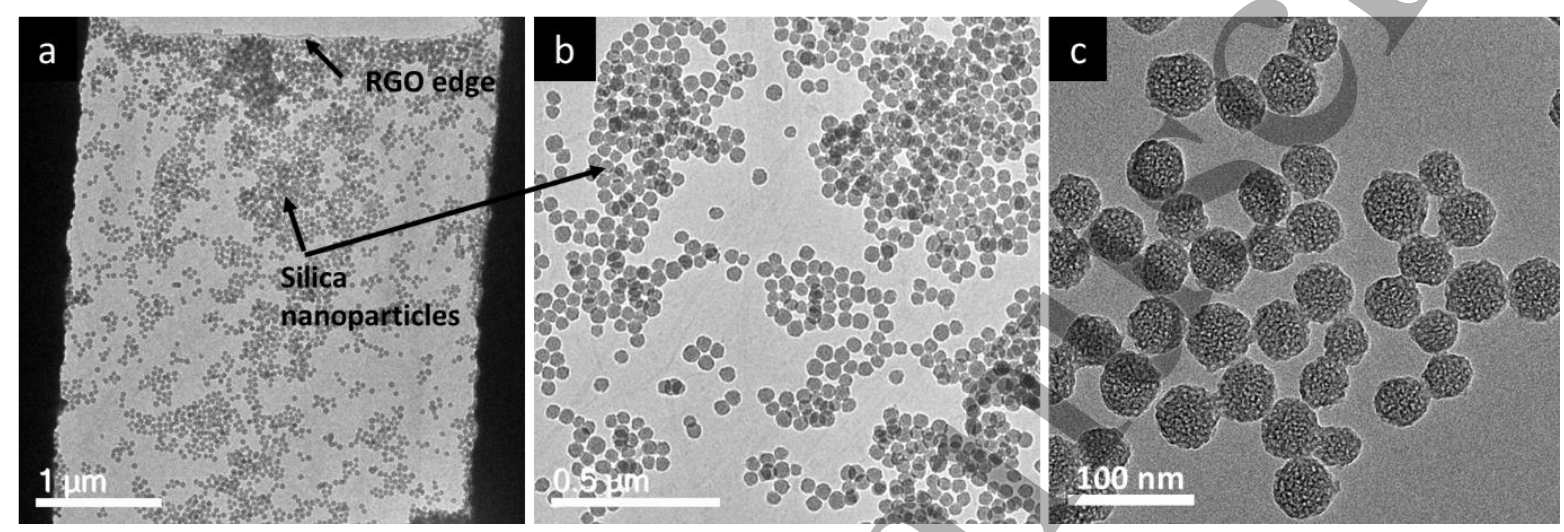

Figure 5 a-c) TEM images of silica nanoparticles supported on the RGO heating membrane at low, medium and high magnifications, respectively.

TEM images of as-prepared silica nanoparticles on the RGO flake are shown in Figures 5a-c. Their diameter ranges between 35 and $50 \mathrm{~nm}$. The silica nanoparticles are uniformly dispersed in the RGO between two electrodes (see Figure 5a). In the high-magnification TEM image (Figure 5c), the porous morphology of the silica nanoparticles can also be observed. Some of the particles are also attached together by Van der Waals forces or surface impurities or functional groups. Both ex-situ and in-situ studies were executed to determine the melting/evaporation temperature of silica nanoparticles.

Similar to the previous case, when the current was ramped through the RGO heater. Many high temperature-induced behaviours of the silica nanoparticles were detected. As soon as the temperature of the RGO increased above $1500{ }^{\circ} \mathrm{C}$, the silica nanoparticles started to agglomerate, as shown in Figure 6. The temperature of the heater rose slowly until the silica nanoparticle started melting and fusing. From panels I and II (Figure 6), it was also clear that a chain-like structure was formed initially. Furthermore, this chain-like structure continuously changed shape due to thermal instability. To minimize surface free energy and maximize surface-to-volume ratio at high temperature, the chain-like structure disintegrated into two spherical particles. Before the particle separation, necking of the chain-like structure was witnessed (see panel IV and V, Figure 6), and silica's porous morphology disappeared. Upon subsequent heating, the size of particles reduced until complete evaporation occurred (panels V-IX, Figure 6). As the maximum temperature developed at the centre of RGO flakes, the particles far from the electrode evaporated first, then close to it. All the particles in (red circle) Figure 6 behaved similarly. Firstly, there is the formation of a chain-like structure, and then disintegration occurs into spherical particles, and finally, evaporation. 


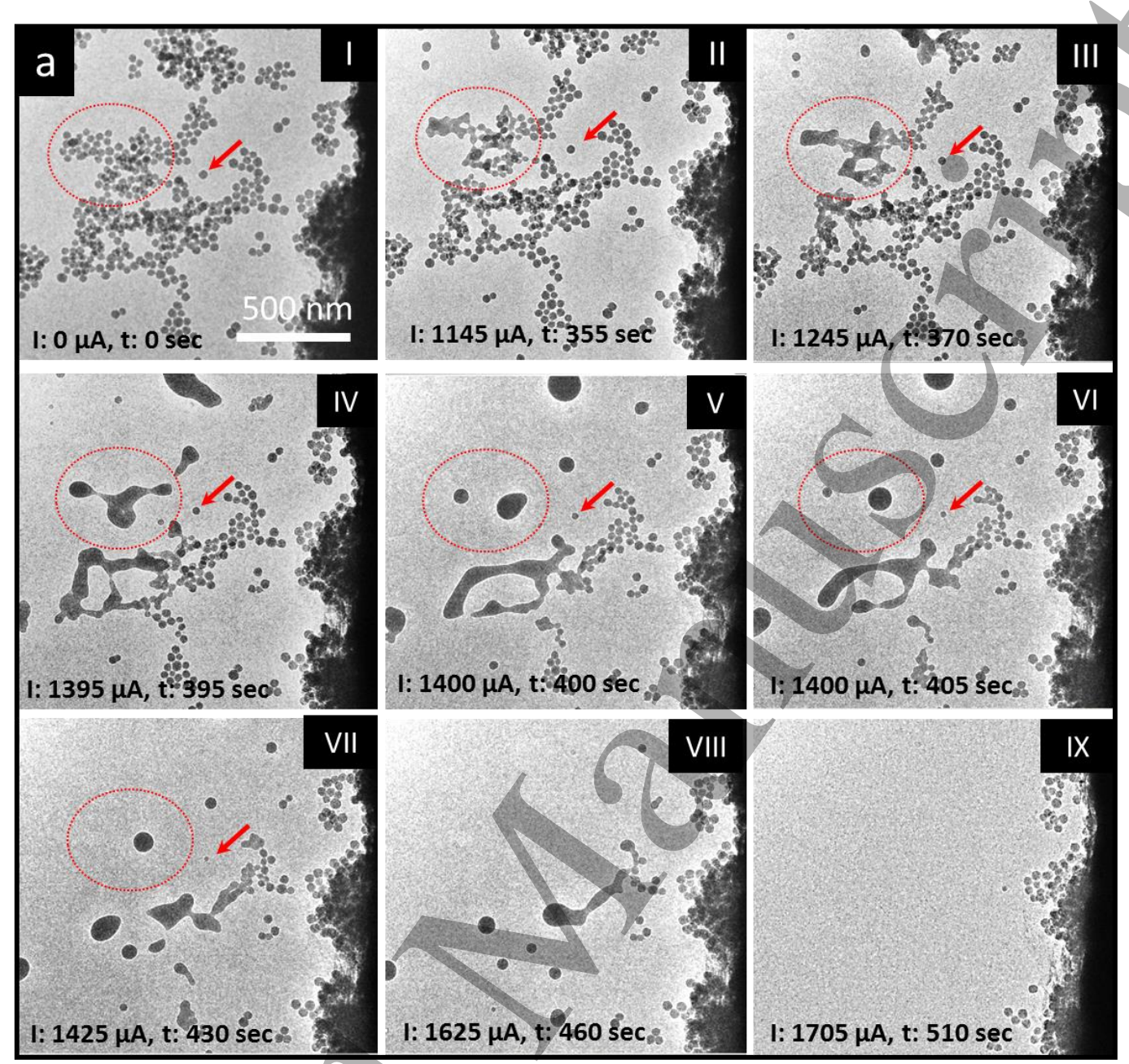

Figure 6 a) Time series TEM images (panel I-IX) of thermal annealing of silica nanoparticles loaded onto the RGO heater.

An isolated silica nanoparticle was also analysed and one of the particles marked by the solid red arrow, as seen in Figure 6. During the heating of nanoparticles, an Isolated silica nanoparticles acted differently from the agglomerated particles. No movement and aggregation of isolated particles was recorded; these might be due to the attachment of nanoparticles with the defects.[7,21,33] However, isolated particles also lost their porous morphology due to surface atoms diffusion at elevated temperature and formed dense sphere as observed by almost $20 \%$ reduction in the size of nanoparticle (see panels III and IV in Figure 6). Due to the structure instability of the isolated nanoparticle close to its melting point, the size of the nanoparticle dropped continuously from $50 \mathrm{~nm}$ to less than $10 \mathrm{~nm}$, as observed in panels I-VII. Finally, this particle disappeared at $\mathrm{t}=460 \mathrm{sec}$, as shown in Figure 6 (panel VIII) due to complete evaporation. Moreover, at the end of the evaporation of silica nanoparticles, we noticed the irregular-shaped pores in the carbon lattice. The pore with different sizes and shapes were recorded, as seen in Figures S7a and S7b. The smallest pore of $18 \mathrm{~nm}$ was observed after the complete evaporation of the isolated particle (see pore marked by the red arrow in Figure S7a).

The ex-situ thermal stability of the silica nanoparticles was inspected by heating the chips inside a vacuum $(<5$ mTorr $)$ tube furnace at $1100{ }^{\circ} \mathrm{C}$. The TEM micrographs were collected from the same 
chip before and after ex-situ heat treatment, as shown in Figures S8a and S8b. The silica nanoparticles were very stable at this temperature, with no melting or fusion of the particles. Moreover, the silica nanoparticles maintained their porous morphology (Figure S8c). Furthermore, thermal gravimetric analysis (TGA) of these nanoparticles was collected between temperatures of $25-1500{ }^{\circ} \mathrm{C}$ (heating rate of $10^{\circ} \mathrm{C} / \mathrm{min}$ ) under Ar flow. Even at a temperature of as high as $1500{ }^{\circ} \mathrm{C}$, a mass loss of less than $5 \%$ was recorded (Figure S8d) due to the removal of organic impurities or absorbed water.[26] These ex-situ results also proved the excellent thermal stability of silica nanoparticles.

\section{Discussion}

In this work, GO-based and RGO-based heaters were developed and used for in situ thermal experiments inside TEM. The electron beam-induced knockout damage on GO flakes was minimized by limiting the accelerating voltage to $80-120 \mathrm{kV}$. We prepared tens of devices from a few microliters of dispersion, and only 1-2 $\mu \mathrm{L}$ was sufficient to craft a single device. In the first step, current-induced Joule heating originated the heating and reduction of GO. Initially, GO had very high electrical resistance brought down to more than $90 \%$ by localized heating. The resistance decreased rapidly with a slight increase in current. With a further rise in current-density of the heater, the resistance decreased gradually and linearly. The current-induced heating led to the removal of functional groups, as reported in the literature.[20,29] Furthermore, with Raman spectroscopy and EELS, the improved carbon content bonded with sp2, and the crystallinity of graphene were established. $\mathrm{I}_{\mathrm{D}} / \mathrm{I}_{\mathrm{G}}$ ratio plunged significantly from 1 to 0.34 , confirming the reduction of GO to RGO. In the EELS spectra, the oxygen peak also vanished completely. Additionally, the thermal investigation of the Au and silica nanoparticles was carried out using the RGO-based heater, as shown in Figure 7. It was found that the RGO heater had radial heat distribution. Moreover, the maximum temperature was achieved in the centre of the suspended RGO flake, while close to the electrode, the temperature was lower due to heat dissipation through metal electrodes to a silicon chip. $[3,12]$

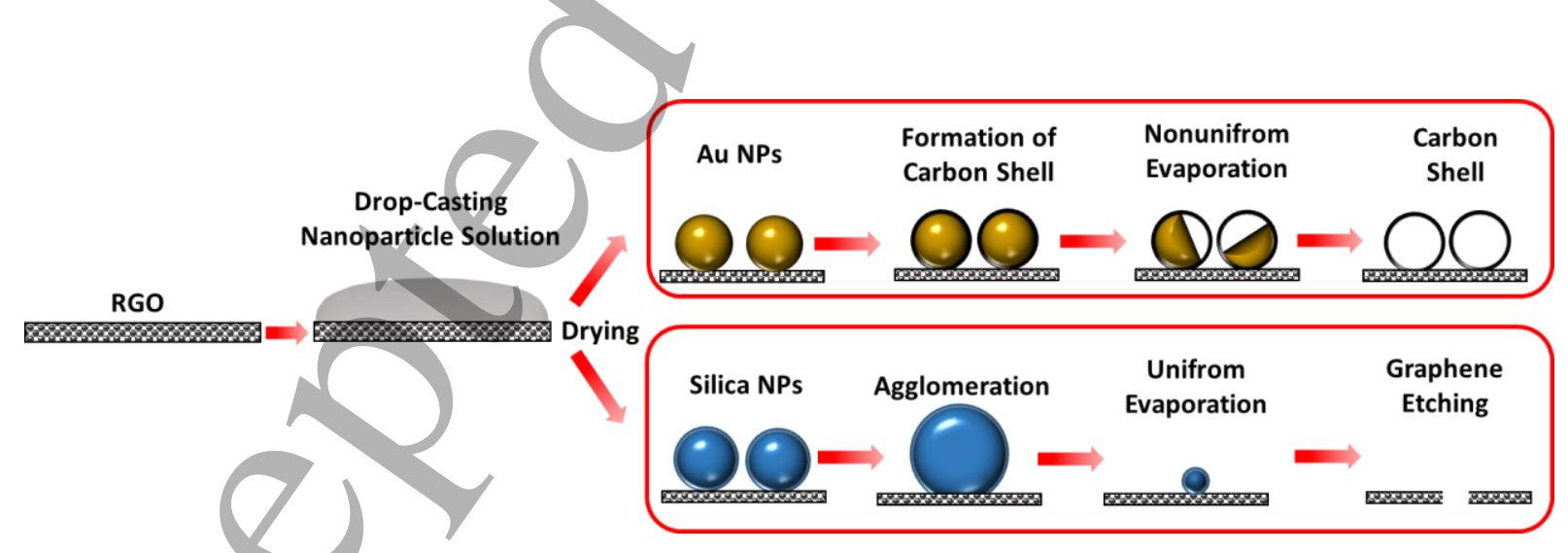

Figure 7 Schematic process flow of in-situ thermal stability investigation of Au and silica nanoparticles.

In order to determine the temperature of the GO heater, we compared the current density of GO heater with the once reported in the literature. As described by Huang et al., the temperature increase in carbon nanotube and graphene layer was around $1700-2000{ }^{\circ} \mathrm{C}$ inside the electron microscope at a current density greater than $1 \times 10^{7} \mathrm{~A} / \mathrm{cm}^{2} .[30,31]$ For complete graphitization of 
the GO flake and melting/evaporation of silica nanoparticles, the current density greater than 1.0 $\mathrm{x} 10^{7} \mathrm{~A} / \mathrm{cm}^{2}$ was used. The Raman and EELS spectra of the GO heater were compared with the electrically or thermally reduced GO samples as reported in the literature; the temperature was around 1500-2400 ${ }^{\circ} \mathrm{C}$ for the graphitization of the amorphous carbon or graphene oxide and forming the ordered structure. The intensity ratio of Raman peaks from $D$ and $G\left(I_{D} / I_{G}\right)$ band decreased to 0.34 for the RGO samples after the current induced reduction. As observed by Song et al., Li et al. and Dez-Betriu et al., to obtain the ID / IG ratio less than 0.35 for thermally reduced graphene oxide samples, the temperature of more than 1700-2000 ${ }^{\circ} \mathrm{C}$ was required.[34-36] Furthermore, Asaka et al. and Barreiro et al. showed the graphitization of amorphous carbon on the surface of multi-walled carbon nanotube and graphene at approximately $2000^{\circ} \mathrm{C}$ and $1600^{\circ}$ $\mathrm{C}$, respectively, during the Joule heating experiment inside TEM.[14,22] These previous research provided good information regarding the temperature rise during the Joule heating experiments.

Upon Joule heating of RGO, nonuniform distribution of heat resulted in melting/evaporation of $\mathrm{Au}$ particles in the centre first and then at the edges of RGO flake. The melting point of the bulk $\mathrm{Au}$ is $1064{ }^{\circ} \mathrm{C}$. The melting point is known to decrease with a reduction in size for nanoparticles.[37] In this work, evaporation of $\mathrm{Au}$ nanoparticles occurred above $950{ }^{\circ} \mathrm{C}$ as confirmed by ex-situ and in-situ studies. In recent work, Liu et al. found that melting / evaporation of prism-shaped Au nanoparticles took place at $875^{\circ} \mathrm{C}$ using commercial heating chips.[38] Hobbs calculations showed that the temperature rise due to electron beam-induced heating is negligible in highly conductive samples.[39]

When nanoparticles were analyzed at high magnification with an aberration corrected microscope, carbon precipitation on crystalline Au particles was detected. In previous studies, similar formation of the carbon nanoshell has been observed for different metal nanoparticles such as $\mathrm{Cu}, \mathrm{Ag}, \mathrm{Au}$ nanoparticles.[40-42] Because of the appearance of a carbon nanoshell around the $\mathrm{Au}$ nanoparticles, no Oswald ripening occurred during the heating experiment. The carbon nanoshells are formed by electron beam-induced disintegration of hydrocarbons molecules and subsequent deposition and graphitization of carbon around the crystalline nanoparticles at elevated temperatures. Sutter et al. studied the interaction of $\mathrm{Au}$ nanoparticles on an amorphous carbon support, and they reported the formation of carbon layers at temperatures as low as $425{ }^{\circ} \mathrm{C}$ subjected to intense electron irradiation.[42] The melting of Au nanoparticles was recorded as observed in Figure 4. Previous studies found that, upon heating, pressures of as high as three GPa were generated due to the encapsulation of nanoparticles inside the graphitic shell.[32] That may be the reason before evaporation started, Au nanoparticles transformed from ordered structures to more disordered ones without significant shape changes.[40] The evaporation of Au nanoparticles began only when the temperature was increased beyond this point. In evaporation, the carbon nanoshell preserved its shape and structure.

As shown in Figure 4, within last $75 \mathrm{sec}$ particles disappeared completely with current rise of 40 $\mu \mathrm{A}$. There were mainly three reasons for the faster evaporation of Au nanoparticles with the same incremental current. As the flake was heated with current-induced heating, the temperature rise was not linear with the current increase, rather it was exponential. As reported in literature, the temperature rose exponentially with increasing bias through of suspended nanomaterials due to Joule heating. $[43,44]$ During our experiments, the temperature of system soared continuously with the current. That was the one reason for the faster disappearance of Au nanoparticles. In the literature, most of the experiments were performed at constant temperature after initiation of 
evaporation/sublimation. Therefore, the rate of evaporation/sublimation of nanoparticle was slower in those cases. [38,41,45]

Another factors that affected the evaporation rate of nanoparticles were size, shape, and defects. Buffat et al. found that the melting point of nanoparticles deceased with the dropping size of particles.[37] Further, Li. et al. observed that the $25 \mathrm{~nm}$ silver nanoparticles took time more than twice of the $15 \mathrm{~nm}$ particles during sublimation at same temperature. Moreover, the sublimation of nanoparticle was slow and linear initially. After $8 \mathrm{~nm}$, the size of particle dropped rapidly as governed by Kelvin equation and kinetic theory.[45] Electron irradiation under ultra-high vacuum of the TEM column also affected the structure, shape and size of nanoparticles as reported by Tanaka et al. and Ajayen et al..[46,47] Tanaka et al. also detailed disappearance of Pd nanoparticles within $15 \mathrm{sec}$ when subjected to strong beam irradiation without external heating.[46] Even though, we tried to minimize the electron beam irradiation for the most of experiments, there might be some effects of electron beam at high magnification. Thus, we believe that combination of above factors might cause the rapid evaporation of Au nanoparticles.

The silica nanoparticles have been explored as catalyst support for many applications. [48,49] Therefore, it is crucial to assess the thermal stability of these nanoparticles at elevated temperatures. Even at a temperature above $1000{ }^{\circ} \mathrm{C}$ these nanoparticles retain their high surface area, chemical properties, morphology, and structure. However, the visual inspection of stability of porous morphology at extreme temperature is missing. In this work, the silica nanoparticles were very stable. They retained the porous morphology when subjected to the ex-situ heattreatment at $1100{ }^{\circ} \mathrm{C}$. The particles are synthesized in extreme chemical conditions and subjected to high-temperature calcination during preparation. No evidence of melting/evaporation was detected, and particles retained their shape, size, and location on the RGO heating membrane. The heat-treatment of the silica nanoparticles on an RGO support did not display the formation of a carbon shell. Moreover, TGA also proved excellent thermal stability of silica particles from room temperature to $1500{ }^{\circ} \mathrm{C}$ with no significate weight loss.[26,50]

This is the first work of in situ TEM observation where the melting/evaporation kinetics of a hightemperature silica nanoparticle was recorded. By joule heating of RGO - silica nanoparticles system to elevated temperature, significant changes in the size and shape of nanoparticles followed and agglomeration of particles initiated. First, silica nanoparticles fused to form an irregular chainlike structure and then transformed into more dense and regular-shaped large particles. Due to Rayleigh's instability of the elongated chain-like structure, the fragmentation into elliptical particles followed.[51] Further, the disintegrated elliptical particles converted into a spherical shape due to surface atom diffusion. The diameter of the silica nanoparticles dropped evenly due to the uniform evaporation from the surface. Contrary to the case for Au nanoparticles, no carbon shell was detected here; instead, the silica nanoparticles etched the graphene support. Few nanometre-sized pores were created in RGO, and this was attributable to a carbothermal reaction between silica and graphene at elevated temperatures. By carbothermal reaction, Martin et al. reported synthesis of $\mathrm{SiC}$ particles from silica and carbon source at $1500-1800{ }^{\circ} \mathrm{C}$ under vacuum. [52] These conditions were similar to observed in our work. The carbothermal reaction between silica and carbon was shown in Figure S7, a small crystalline particle was detected at the edge of the hole after the reaction. The spectroscopical and structural analyses of the crystalline particles were not performed because they were out of the scope of this work. In previous reports, nonmetallic and metallic nanoparticles induced catalytic hydrogenation or oxidation caused the 
formation of pores in graphene. [53-55] In our experiments, catalytic hydrogenation and oxidation were not possible due to the absence of gases in the TEM vacuum chamber.

It was unclear what caused the uniform and nonuniform evaporation in silica and Au nanoparticles respectively, inside the ultra-high vacuum of the TEM column at elevated temperature. As reported in the literature, the size, shape, orientation, and surface defects profoundly affect the degree and uniformity of evaporation. The divergent evaporation behaviour of $\mathrm{Au}$ and Silica nanoparticles was strongly related to the difference in chemical nature and surface morphology.[45,56,57] Moreover, no agglomeration of Au nanoparticles was recorded before melting/evaporation. In the case of silica nanoparticles, significant morphological changes were noticed: aggregation, fragmentation, and formation of spherical particles followed by melting/evaporation and etching of carbon lattice.

\section{Conclusions}

Here, we demonstrated a simple and cost-effective method to fabricate GO/RGO-based in-situ TEM heater. The heater was manufactured by simply drop-casting GO dispersion onto the prepatterned Si chips. The GO/RGO-based heater has several advantages: low cost, extensive heating range (R.T.- $1700{ }^{\circ} \mathrm{C}$ ), electron transparent membrane, good mechanical and thermal stability, and ability to resolve atomically defined images. The GO support was successfully reduced using Joule heating and was subsequently used for heating experiments. Moreover, we investigated the stability of $\mathrm{Au}$ and silica nanoparticles at temperatures higher than their melting points. Au nanoparticles being crystalline materials showed nonuniform evaporation while noncrystalline silica nanoparticles evaporated uniformly. Their interaction with carbon also varied greatly, and one stabilized the carbon shell while the other predatorily etched the RGO support. The temperature of suspended RGO was raised to more than $1700^{\circ} \mathrm{C}$ as confirmed by spectroscopically and evaporation of metallic and non-metallic nanoparticles. The following methods can be used to analyse a large variety of nanomaterials and devices, are excellent alternatives to the commercial and graphene-based heater that can be cleaned quickly and reused.

\section{Conflicts Of Interest}

There are no conflicts to declare.

\section{Acknowledgments}

NMB and DM thank KAUST for graduate scholarships. This project was supported by KAUST (BAS/1/1346-01-01/02). We acknowledge the technical support provided by Dalaver and others scientist from Imaging and Characterization core labs at KAUST.

\section{References}

[1] Golberg D, Costa P M F J, Mitome M, Hampel S, Haase D, Mueller C, Leonhardt A and Bando Y 2007 Copper-Filled Carbon Nanotubes: Rheostatlike Behavior and Femtogram Copper Mass Transport Advanced Materials 19 1937-42 
[2] Costa P M F J, Cachim P B, Gautam U K, Bando Y and Golberg D 2009 The mechanical response of turbostratic carbon nanotubes filled with Ga-doped $\mathrm{ZnS}$ : I. Data processing for the extraction of the elastic modulus Nanotechnology 20405706

[3] Costa P M F J, Gautam U, Bando Y and Golberg D 2011 The electrical delivery of a sublimable II-VI compound by vapor transport in carbon nanotubes Carbon 49 3747-54

[4] Batra N M, Syed A and Costa P M F J 2019 Current-induced restructuring in bent silver nanowires Nanoscale 11 3606-18

[5] Costa P M F J, Gautam U K, Wang M, Bando Y and Golberg D 2009 Effect of crystalline filling on the mechanical response of carbon nanotubes Carbon 47 541-4

[6] Blech I A and Meieran E S 1967 Direct transmission electron microscope observation of electrotransport in aluminum thin films Appl. Phys. Lett. $11263-6$

[7] Palanisamy T, Alazmi A, Batra N M and Costa P MF J 2021 An in-situ assessment of postsynthesis thermal annealing of platinum nanoparticles supported on graphene Materials Science and Engineering: B 272115370

[8] Hou Z, Zhang Q, Zhang X, Xu G, Xia J, Ding B, Li H, Zhang S, Batra N M, Costa P M F J, Liu E, Wu G, Ezawa M, Liu X, Zhou Y, Zhang X and Wang W 2020 Current-Induced Helicity Reversal of a Single Skyrmionic Bubble Chain in a Nanostructured Frustrated Magnet Advanced Materials 321904815

[9] Allard L F, Bigelow W C, Jose-Yacaman M, Nackashi D P, Damiano J and Mick S E 2009 A new MEMS-based system for ultra-high-resolution imaging at elevated temperatures Microsc. Res. Tech. 72 208-15

[10] Grant A W, Hu Q-H and Kasemo B 2004 Transmission electron microscopy windows for nanofabricated structures Nanotechnology 15 1175-81

[11] Westenfelder B, Meyer J C, Biskupek J, Kurasch S, Scholz F, Krill 3rd C E and Kaiser U 2011 Transformations of carbon adsorbates on graphene substrates under extreme heat Nano Lett $115123-7$

[12] Westenfelder B, Meyer J C, Biskupek J, Algara-Siller G, Lechner L G, Kusterer J, Kaiser U, Krill C E, Kohn E and Scholz F 2011 Graphene-based sample supports for in situ highresolution TEM electrical investigations J. Phys. D: Appl. Phys. 44055502

[13] Yuk J M, Park J, Ercius P, Kim K, Hellebusch D J, Crommie M F, Lee J Y, Zettl A and Alivisatos A P 2012 High-Resolution EM of Colloidal Nanocrystal Growth Using Graphene Liquid Cells Science 336 61-4

[14] Barreiro A, Börrnert F, Avdoshenko S M, Rellinghaus B, Cuniberti G, Rümmeli M H and Vandersypen L M K 2013 Understanding the catalyst-free transformation of amorphous carbon into graphene by current-induced annealing Scientific Reports $\mathbf{3}$ 
[15] Erni R, Rossell M D, Nguyen M-T, Blankenburg S, Passerone D, Hartel P, Alem N, Erickson K, Gannett W and Zettl A 2010 Stability and dynamics of small molecules trapped on graphene Physical Review B 82165443

[16] Zhang J, Lin L, Sun L, Huang Y, Koh A L, Dang W, Yin J, Wang M, Tan C, Li T, Tan Z, Liu Z and Peng H 2017 Clean Transfer of Large Graphene Single Crystals for High-Intactnéss Suspended Membranes and Liquid Cells Adv. Mater. 291700639

[17] Kirilenko D A, Dideykin A T, Aleksenskiy A E, Sitnikova A A, Konnikov S G and Vul' A Ya 2015 One-step synthesis of a suspended ultrathin graphene oxide film: Application in transmission electron microscopy Micron 68 23-6

[18] Xu Z, Bando Y, Liu L, Wang W, Bai X and Golberg D 2011 Electrical Conductivity, Chemistry, and Bonding Alternations under Graphene Oxide to Graphene Transition As Revealed by In Situ TEM ACS Nano 5 4401-6

[19] Pelaez-Fernandez M, Bermejo A, Benito A M, Maser W K and Arenal R 2021 Detailed thermal reduction analyses of graphene oxide via in-situ TÉM/EELS studies Carbon 178 477-87

[20] Go'mez-Navarro C, Weitz R T, Bittner A M, Scolari M, Mews A, Burghard M and Kern K 2007 Electronic Transport Properties of Individual Chemically Reduced Graphene Oxide Sheets nano Letters 7 3499-503

[21] Wei Z, Wang D, Kim S, Kim S-Y, Hu Y, Yakes M K, Laracuente A R, Dai Z, Marder S R, Berger C, King W P, de Heer W A, Sheehan P E and Riedo E 2010 Nanoscale Tunable Reduction of Graphene Oxide for Graphene Electronics Science 328 1373-6

[22] Asaka K, Karita M and Saito Y 2011 Graphitization of amorphous carbon on a multiwall carbon nanotube surface by catalyst-free heating Appl. Phys. Lett. 99091907

[23] Mahalingam D K, Falca G, Upadhya L, Abou-Hamad E, Batra N, Wang S, Musteata V, da Costa P M and Nunes S P 2020 Spray-coated graphene oxide hollow fibers for nanofiltration Journal of Membrane Science $\mathbf{6 0 6} 118006$

[24] Mahalingam D K, Wang S and Nunes S P 2018 Graphene Oxide Liquid Crystal Membranes in Protic Ionic Liquid for Nanofiltration ACS Appl. Nano Mater. 1 4661-70

[25] Nitin M. Batra P M C 2018 Membraneless platform for correlated analysis of nanomaterials

[26] Polshettiwar V, Cha D, Zhang X and Basset J M 2010 High-surface-area silica nanospheres (KCC-1) with a fibrous morphology Angew Chem Int Ed Engl 49 9652-6

[27] Mahjouri-Samani M, Zhou Y S, Xiong W, Gao Y, Mitchell M and Lu Y F 2009 Laser induced selective removal of metallic carbon nanotubes Nanotechnology $\mathbf{2 0} 495202$ 
[28] Batra N M, Patole S P, Abdelkader A, Anjum D H, Deepak F L and Costa P M F J 2015 Structural changes of electron and ion beam-deposited contacts in annealed carbon-based electrical devices Nanotechnology 26445301

[29] Yao Y, Fu K K, Yan C, Dai J, Chen Y, Wang Y, Zhang B, Hitz E and Hu L 2016 ThreeDimensional Printable High-Temperature and High-Rate Heaters ACS Nano $105272-9$

[30] Huang J Y, Chen S, Jo S H, Wang Z, Han D X, Chen G, Dresselhaus M S and Ren Z F 2005 Atomic-scale imaging of wall-by-wall breakdown and concurrent transport measurements in multiwall carbon nanotubes Phys Rev Lett 94236802

[31] Huang J Y, Ding F, Yakobson B I, Lu P, Qi L and Li J 2009 In situ observation of graphene sublimation and multi-layer edge reconstructions Proc Natl Acad Sci U S A 106 10103-8

[32] Banhart F, Hernandez E and Terrones M 2003 Extreme superheáting and supercooling of encapsulated metals in fullerenelike shells Phys Rev Lett 90185502

[33] Alazmi A, El Tall O, Rasul S, Hedhili M N, Patole S P and Costa P M F J 2016 A process to enhance the specific surface area and capacitance of hydrothermally reduced graphene oxide Nanoscale 8 17782-7

[34] Song N, Lu C, Chen C, Ma C and Kong Q 2017 Effect of annealing temperature on the mechanical properties of flexible graphene films New Carbon Materials 32 221-6

[35] Li T, Pickel A D, Yao Y, Chen Y, Zeng Y, Lacey S D, Li Y, Wang Y, Dai J, Wang Y, Yang B, Fuhrer M S, Marconnet A, Dames C, Drew D H and Hu L 2018 Thermoelectric properties and performance of flexible reduced graphene oxide films up to 3,000 K Nature Energy 3 $148-56$

[36] Díez-Betriu X, Álvarez-García S, Botas C, Álvarez P, Sánchez-Marcos J, Prieto C, Menéndez R and de Andrés A 2013 Raman spectroscopy for the study of reduction mechanisms and optimization of conductivity in graphene oxide thin films J. Mater. Chem. C 1 6905-12

[37] Buffat P and Borel J P 1976 Size effect on the melting temperature of gold particles Physical Review A 13 2287-98

[38] Liu Y, Yuan H, Wang H and Wang Z 2021 In Situ Transmission Electron Microscopy Investigation of Melting/Evaporation Kinetics in Anisotropic Gold Nanoparticles Materials 147332

[39] Hobbs L W 1979 Radiation effects in analysis of inorganic specimens by TEM

[40] Chen C, Hu Z, Li Y, Liu L, Mori H and Wang Z 2016 In-Situ High-Resolution Transmission Electron Microscopy Investigation of Overheating of Cu Nanoparticles Sci Rep 619545

[41] Asoro M A, Kovar D and Ferreira P J 2013 In situ Transmission Electron Microscopy Observations of Sublimation in Silver Nanoparticles ACS Nano 7 7844-52 
[42] Sutter E, Sutter P and Zhu Y 2005 Assembly and Interaction of Au/C Core-Shell Nanostructures: In Situ Observation in the Transmission Electron Microscope Nano Letters $52092-6$

[43] Park J, Han D, Choi S, Kim Y and Kwak J 2019 Flexible transparent film heaters using a ternary composite of silver nanowire, conducting polymer, and conductive oxide RSC Ady. 9 $5731-7$

[44] Choi J H, Shin D H, Inani H, Kwon M H, Mustonen K, Mangler C, Park M, Jeong H, Lee D S, Kotakoski J and Lee S W 2020 Transformation and Evaporation of Surface Adsorbents on a Graphene "Hot Plate" ACS Appl. Mater. Interfaces 12 26313-9

[45] Li J, Wang Z, Li Y and Deepak F L 2019 In Situ Atomic-Scale Observation of Kinetic Pathways of Sublimation in Silver Nanoparticles Advanced Science 1802131

[46] Tanaka M, Takeguchi M and Furuya K 2002 Behavior of metal nanoparticles in the electron beam Micron 33 441-6

[47] Ajayan P M and Marks L D 1989 Experimental evidence for quasimelting in small particles Phys. Rev. Lett. 63 279-82

[48] Shahul Hamid M Y, Triwahyono S, Jalil A A, Che Jusoh N W, Izan S M and Tuan Abdullah T A 2018 Tailoring the Properties of Metal Oxide Loaded/KCC-1 toward a Different Mechanism of CO2 Methanation by in Situ IR and ESR Inorg Chem 57 5859-69

[49] Werghi B, Pump E, Tretiakov M, Abou-Hamad E, Gurinov A, Doggali P, Anjum D H, Cavallo L, Bendjeriou-Sedjerari A and Basset J M 2018 Exploiting the interactions between the ruthenium Hoveyda-Grubbs catalyst and Al-modified mesoporous silica: the case of SBA15 vs. KCC-1 Chem Sci $93531-7$

[50] Bouhrara M, Ranga C, Fihri A, Shaikh R R, Sarawade P, Emwas A-H, Hedhili M N and Polshettiwar V 2013 Nitridated Fibrous Silica (KCC-1) as a Sustainable Solid Base Nanocatalyst ACS Sustainable Chemistry \& Engineering 1 1192-9

[51] Toimil Molares M E, Balogh A G, Cornelius T W, Neumann R and Trautmann C 2004 Fragmentation of nanowires driven by Rayleigh instability Appl. Phys. Lett. 85 5337-9

[52] Martin H-P, Ecke R and Müller E 1998 Synthesis of nanocrystalline silicon carbide powder by carbothermal reduction Journal of the European Ceramic Society 18 1737-42

[53] Severin N, Kirstein S, Sokolov I M and Rabe J P 2009 Rapid Trench Channeling of Graphenes with Catalytic Silver Nanoparticles Nano Lett. 9 457-61

[54] Gao L, Ren W, Liu B, Wu Z-S, Jiang C and Cheng H-M 2009 Crystallographic Tailoring of Graphene by Nonmetal SiO $x$ Nanoparticles J. Am. Chem. Soc. 131 13934-6 
[55] Booth T J, Pizzocchero F, Andersen H, Hansen T W, Wagner J B, Jinschek J R, DuninBorkowski R E, Hansen O and Bøggild P 2011 Discrete Dynamics of Nanoparticle Channelling in Suspended Graphene Nano Lett. 11 2689-92

[56] Nanda K K, Kruis F E and Fissan H 2002 Evaporation of Free PbS Nanoparticles: Evidence of the Kelvin Effect Phys. Rev. Lett. 89256103

[57] Sambles J R, Skinner L M and Lisgarten N D 1970 An electron microscope study of evaporating small particles: the Kelvin equation for liquid lead and the mean surface energy of solid silver Proc. R. Soc. Lond. A 318 507-22 